\title{
Cluster analysis of preoperative echocardiographic findings and outcomes following left ventricular device implantation
}

\author{
Paul C. Tang, MD, PhD, ${ }^{a}$ Jonathan W. Haft, MD, ${ }^{a}$ Matthew A. Romano, MD, ${ }^{a}$ Abbas Bitar, MD, \\ Reema Hasan, MD, ${ }^{\mathrm{b}}$ Maryse Palardy, MD, ${ }^{\mathrm{b}}$ Xiaoting $\mathrm{Wu}, \mathrm{PhD},{ }^{\mathrm{a}}$ Keith Aaronson, $\mathrm{MD},{ }^{\mathrm{b}}$ and \\ Francis D. Pagani, MD, $\mathrm{PhD}^{\mathrm{a}}$
}

\section{ABSTRACT}

Objective: To investigate whether preoperative echocardiography findings determine postoperative continuous-flow left ventricular assist device outcomes.

Methods: From January 2003 to June 2017, 490 patients received a durable, continuous-flow left ventricular assist device. Two-step clustering of parameters including heart rate and preoperative echocardiographic findings (ie, left ventricular [LV] ejection fraction, right ventricular [RV] function, aortic insufficiency, mitral regurgitation [MR], tricuspid regurgitation [TR]) was performed and identified 5 distinct clusters associated with LV failure: group 1: moderate right ventricular dysfunction (RVD), severe MR and mild TR $(n=110)$; group 2: severe RVD, severe MR and TR $(n=64)$; group 3: moderate RVD and severe aortic insufficiency $(\mathrm{n}=16)$; group 4: mild RVD and mild valvular pathology $(\mathrm{n}=163)$; and group 5: moderate-severe RVD and mild valvular pathology $(n=137)$. Silhouette measure of cohesion and separation demonstrated satisfactory separation at 0.6 .

Results: Group 2 had the greatest Interagency Registry for Mechanically Assisted Circulatory Support Level $1(25 \%, P=.010)$, preoperative right atrial pressure $(11 \pm 5 \mathrm{~mm} \mathrm{Hg}, P<.001)$, incidence of postoperative right ventricular failure (RVF; $20 \%, P=.001)$, delayed closure of the sternum $(61 \%, P=.002)$, postoperative permanent dialysis $(6 \%, P=.04)$, rate of tricuspid valve repair $(\mathrm{n}=52$; $81 \%, P<.001)$, and lowest RV stroke work index $\left(489 \pm 228 \mathrm{cc} \mathrm{mm} \mathrm{Hg} / \mathrm{m}^{2} /\right.$ beat, $P<.001)$. RVF in groups $1,3,4$, and 5 was $6 \%, 0 \%, 4 \%$, and $9 \%$, respectively. No differences in incidence of heart transplantation $(P=.400)$ or survival $(P=.535)$ were found. Severe TR predicted RVF in those with moderatesevere preoperative $\operatorname{RVD}(P=.001$, odds ratio 3.9$)$.

Conclusions: Clustering demonstrated the importance of preoperative TR in predicting RVF. Combined severe LV and RV failure with severe MR and TR portends the worse prognosis. (J Thorac Cardiovasc Surg 2019;157:1851-60)

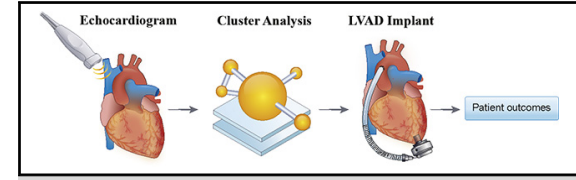

Cluster groups of pre-cfLVAD echocardiographic findings associated with surgical outcome.

\section{Central Message}

Atrioventricular valve insufficiency contributes to heart failure after cfLVAD. Pharmacologic and mechanical support may be expected.

\section{Perspective}

Cluster analysis defined severe biventricular dysfunction with severe regurgitation of both atrioventricular valves as highly predictive of right heart failure post-cfVLAD compared with lesser degrees of valve pathology. In addition to ventricular function, dual valvular regurgitation in series has important implications. This knowledge can promote early use of pharmacologic and/or mechanical support.

See Commentaries on pages 1861 and 1863.
Because of the shortage of suitable donor hearts for transplantation, durable mechanical circulatory support devices

\footnotetext{
From the ${ }^{\mathrm{a}}$ Department of Cardiac Surgery and ${ }^{\mathrm{b}}$ Division of Cardiovascular Medicine, University of Michigan Frankel Cardiovascular Center, Ann Arbor, Mich.

Received for publication June 6, 2018; revisions received Oct 23, 2018; accepted for publication Nov 14, 2018; available ahead of print Feb 7, 2019.

Address for reprints: Paul C. Tang, MD, PhD, University of Michigan Frankel Cardiovascular Center, 5158 Cardiovascular Center, SPC 5864, 1500 E. Medical Center Drive, Ann Arbor, MI 48109-5864 (E-mail: tangpaul@med.umich.edu). 0022-5223/\$0.00

Published by Elsevier Inc. on behalf of The American Association for Thoracic Surgery

https://doi.org/10.1016/j.jtcvs.2018.11.099
}

have become the prevailing surgical treatment for medically refractive advanced heart failure. An important challenge to successful durable, continuous-flow left ventricular assist device (cfLVAD) therapy is postoperative right ventricular failure (RVF), which occurs in $29.8 \%$ to $38.5 \%$ of patients

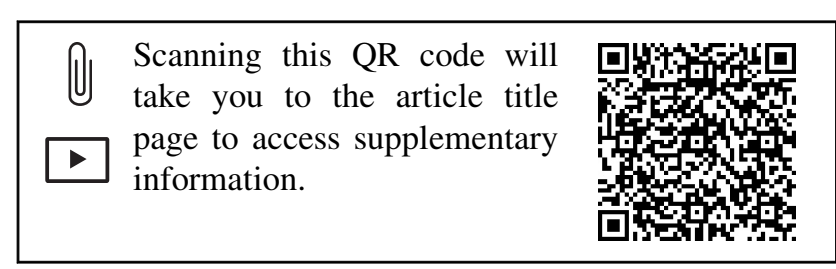




$\begin{aligned} \text { Abbreviations and } & \text { Acronyms } \\ \text { AI } & \text { aortic insufficiency } \\ & =\text { aortic valve } \\ \text { AV } & \text { continuous-flow left ventricular } \\ \text { cfLVAD } & \text { assist device } \\ & \text { Mechanically Assisted Circulatory } \\ \text { INTERMACS }= & \text { Interagency Registry for } \\ & \text { Support } \\ \text { LV } & =\text { left ventricle/ventricular } \\ \text { LVAD } & =\text { left ventricular assist device } \\ \text { MR } & =\text { mitral regurgitation } \\ \text { OR } & =\text { odds ratio } \\ \text { PVR } & =\text { pulmonary vascular resistance } \\ \text { RV } & =\text { right ventricle } \\ \text { RVAD } & =\text { right ventricular assist device } \\ \text { RVD } & =\text { right ventricular dysfunction } \\ \text { RVF } & =\text { right ventricular failure } \\ \text { RVSWI } & =\text { right ventricular stroke work index } \\ \text { TR } & =\text { tricuspid regurgitation } \\ \text { TV } & =\text { tricuspid valve }\end{aligned}$

following implant. ${ }^{1,2}$ RVF is associated with increased complications such as multiorgan failure, postoperative bleeding, poor oxygenation, as well as thromboembolic issues. ${ }^{3}$ Severe RVF requiring right ventricular assist device (RVAD) support leads to greater hospital mortality, and even if successful RVAD weaning occurs, these patients are still impacted by a greater incidence of subsequent heart failure. ${ }^{4}$ Although RVF risk-prediction models have been developed to facilitate patient selection for left ventricular assist device (LVAD) therapy, ${ }^{5}$ these models are only modestly sensitive in identifying postoperative RVF, with a $60 \%$ positive predictive value based on several wellestablished prediction score paradigms. ${ }^{6}$

Although many patients with heart failure have associated single or multivalvular heart disease, ${ }^{7}$ the large majority of studies have focused on single valvular lesions when evaluating their impact on postoperative outcomes, including the effects on right ventricular (RV) function. RVF pathophysiology post-cfLVAD implant is very complex and unlikely to be fully explained by a single valvular lesion. Recent studies have refocused on the impact of mitral regurgitation (MR) on RVF in the setting of LVAD implantation. Taghavi and colleagues ${ }^{8}$ observed that mitral valve surgery at the time of LVAD implant leads to a greater decrement in mean pulmonary artery pressures and pulmonary vascular resistance (PVR) compared with cfLVAD implantation without concomitant valve repair. Cowger and colleagues $^{9}$ also described aortic insufficiency (AI) in patients with cfLVAD can worsen MR and adversely impacting RV function.
This study uses an unsupervised statistical clustering technique without pre-existing investigator biases to categorize preoperative LVAD echocardiographic assessment of valvular disease, ventricular contractility, and heart rate. We hypothesize that multivalvular disease increases the risk of RVF following LVAD implantation.

\section{PATIENTS AND METHODS \\ Patients}

This study with a waiver of informed consent was approved by the University of Michigan institutional review board (institutional review board no. HUM00135533). We conducted a retrospective review of prospectively collected data from the University of Michigan Mechanical Circulatory Support Registry (institutional review board no. HUM00020274) on 490 consecutive patients who underwent durable cfLVAD implantation from January 1, 2003, to June 1, 2017. RVF was defined as (1) a central venous pressure $>18 \mathrm{~mm} \mathrm{Hg}$ with a cardiac index $<2.0 \mathrm{~L} / \mathrm{min} / \mathrm{m}^{2}$ without elevation of pulmonary capillary wedge pressure $>18 \mathrm{~mm} \mathrm{Hg}$; or (2) requirement for an RVAD; or (3) requirement for pulmonary vasodilator (eg, inhaled nitric oxide) or inotrope therapy for $>1$ week following LVAD implantation; in the absence of tamponade, ventricular arrhythmias, or pneumothorax. ${ }^{10}$ Only postoperative RVF occurring during the index hospitalization following cfLVAD implantation was considered in the primary endpoint. The decision for using a RVAD was determined by the need for high-dose inotropes and vasopressors for hemodynamic support and/or when maximal pharmacologic circulatory support was reached without maintaining adequate hemodynamics for peripheral perfusion as a result of RVF.

\section{Follow-up}

Survival data were available for all 490 patients who underwent cfLVAD implantation and obtained through detailed clinical follow-up in the Mechanical Circulatory Support Registry and medical records. Patients were censored at the time of heart transplantation, device explant for cardiac recovery, device de-activation (without replacement), or for transfer of care to another institution. Longest follow-up was 12.8 years, with a total follow up of 998.0 patient years. Mean follow up was $2.04 \pm 2.11$ years with a median follow up of 1.31 (interquartile range 2.24) years. Preoperative echocardiographic assessment was obtained within 30 days of cfLVAD implantation. Right ventricular dysfunction (RVD) was graded as follows: 0 - normal, 1 - mild, 2 - moderate, and 3 - severe RVD. Tricuspid regurgitation (TR), MR, and AI was graded as: 0 - none, 1 trace, 2 - mild, 3 - moderate, and 4 - severe. The primary end point was postoperative RVF. Secondary end points included operative mortality, readmissions, stroke, as well as the combined end point of LVAD survival and survival to heart transplant.

\section{Statistical Methods}

The Pearson $\chi^{2}$ test or Fisher exact test along with binary comparisons was used to analyze categorical variables. An independent Student $t$ test was used to compare continuous variables. Analysis of variance with post hoc Tukey testing or the Kruskal-Wallis method with post hoc Dunn-Bonferroni testing was performed for comparison of continuous variables across multiple groups. Logistic regression was performed to determine the relationship between RVF with TR, MR, and AI severity and degree of RVD. Kaplan-Meier survival analysis with Mantel-Cox statistics was used to analyze survival data. A 2-step cluster analysis was performed that used independent variables in the algorithm consisting of LV ejection fraction, RVD, AI, MR, TR, and heart rate. Using the log-likelihood distance, small subclusters were formulated from the cases by building a modified cluster feature tree to group similar cases together in nodes based on threshold distance as determined by variable mean and variance. The 
second step then further aggregated the previously determined subclusters using Bayesian hierarchical clustering. The final optimal number of clusters was then determined by comparing the minimum intercluster distance among the hierarchically defined cluster possibilities with acceptance of the one with the widest separation. ${ }^{11-14}$ All statistics were performed using Statistical Package for the Social Sciences software (SPSS Inc, version 24, Chicago, Ill).

\section{RESULTS}

\section{Study Population Characteristics}

Study population characteristics are presented in Table E1. These characteristics were consistent with patients with advanced heart failure. The majority of study population was male $(78.4 \%)$ with a mean age of $55.2 \pm 13.2$ years. Baseline hemodynamics included a mixed venous oxygen saturation of $55.6 \pm 8.8 \%$, cardiac index of $2.3 \pm 0.6 \mathrm{~L} / \mathrm{min} / \mathrm{m}^{2}$, and right ventricular stroke work index (RVSWI) of $599.3 \pm 260.0 \mathrm{~g} / \mathrm{m} / \mathrm{m}^{2} /$ beat (Table E2). Baseline echocardiography data demonstrated an LV ejection fraction of $15.4 \pm 5.7 \%$, moderate-severe RVF in $52.0 \%$, severe $\mathrm{AI}$ in $3.3 \%$, severe MR in $33.3 \%$, severe TR in $13.7 \%$, and a mean heart rate of $88.42 \pm 17.76$ (Table E2).

\section{Unsupervised Statistical Categorization of \\ Preoperative Echocardiographic Parameters}

Of the 495 patients who had cfLVAD implantation in the registry, 490 patients had complete echocardiographic data to be included in our cluster analysis. Two-step cluster analysis resulted in 5 distinct groups of echocardiographic findings (Table 1) with an excellent degree of separation ( silhouette cluster of cohesion and separation $=0.6$ ), indicating that the cluster groups are distinct. Characteristics of the categories included left ventricular (LV) failure with group 1: moderate RVD, severe MR, and mild TR ( $\mathrm{n}=110$ ); group 2: severe RVD, severe $\mathrm{MR}$ and TR ( $\mathrm{n}=64)$; group 3: moderate RVD, moderate MR, mildmoderate TR, and severe AI $(n=16)$; group 4: mild RVD and mild valvular pathology $(\mathrm{n}=163)$; and group 5: moderate-severe RVD and mild valvular pathology $(n=137)$. A video discussion of the individual clusters is included with this article (Video 1).

\section{Association of Cluster Grouping and Pre-cfLVAD Acuity of Illness}

Whereas group 2 (biventricular failure and atrioventricular valve regurgitation) had the greatest proportion of patients assigned to Interagency Registry for Mechanically Assisted Circulatory Support (INTERMACS) level 1 profile $(25.0 \%, P=.090)$, group 3 (with severe $\mathrm{AI}$ ) had the greatest number of patients demonstrating progressive hemodynamic decline on inotropes and assigned to INTERMACS level $2(37.5 \%)$. Patients in 2 group 2 largely consisted of those receiving device support for bridge to heart transplant indication $(78.1 \%, P=.002$, Table 2$)$, and was also the youngest group with the lowest incidence of hypertension, diabetes, and hyperlipidemia $(P<.05$, Table 3$)$. Groups 2 and 5 with biventricular failure had the greatest requirement for preoperative temporary circulatory support at $14.1 \%$ and $14.6 \%$, respectively (Table $2, P=.012$ ), and the greatest heart rate at $93.9 \pm 19.9 \mathrm{bpm}$ and $94.2 \pm 17.7 \mathrm{bpm}$, respectively $(P<.001)$. No difference in the frequency or duration of intra-aortic balloon pump use was demonstrated between the groups $(P>.05$, Table 2$)$. Preoperative (Table 4$)$ right atrial pressure was greatest in group $2(P<.001)$ accompanied by the lowest RVSWI $(P=.002)$, and the lowest mixed venous oxygen saturation $(P=.023)$. Group 2 (Table 4$)$ also had a greater alkaline phosphatase level $(P=.094)$ and the greatest total bilirubin $(P<.001)$, suggesting this group has the worse hepatic dysfunction and greater degree of preoperative hypoperfusion.

\section{Operative Features}

As expected, the majority of aortic valve (AV) interventions during cfLVAD implantation were performed in group $3(100 \%, P<.001)$, and this group accounted for the longest cardiopulmonary bypass times $(123.8 \pm 35.1 \mathrm{mi}-$ nutes, $P<.001$ ). Most of the tricuspid valve (TV) procedures were performed in groups 2 and $3(81.3 \%$ and $62.5 \%$, respectively, $P<.001$ ), which displayed the greatest incidence of baseline severe TR. Mitral valve interventions only occurred in 1 patient in our series. AV intervention included AV replacement $(25 \%, \mathrm{n}=4 / 16)$ and repair with the Park stitch $(75 \%, n=12 / 16)$. All tricuspid valve

TABLE 1. Segregated groups using unsupervised cluster analyses

\begin{tabular}{|c|c|c|c|c|c|c|}
\hline Cluster & $1(n=110)$ & $2(n=64)$ & $3(n=16)$ & $4(n=163)$ & $5(n=137)$ & $P$ value \\
\hline LVEF, \% & $14.52 \pm 4.77$ & $13.52 \pm 4.75$ & $13.44 \pm 5.98$ & $17.60 \pm 6.80$ & $14.43 \pm 4.54$ & $<.001$ \\
\hline Moderate-severe RVD & $59(53.6 \%)$ & $51(79.7 \%)$ & $10(62.5 \%)$ & $7(4.3 \%)$ & $128(93.4 \%)$ & $<.001$ \\
\hline Severe AI & $0(0 \%)$ & $0(0 \%)$ & $16(100 \%)$ & $0(0 \%)$ & $0(0 \%)$ & $<.001$ \\
\hline Severe MR & $110(100 \%)$ & $45(70.3 \%)$ & $6(37.5 \%)$ & $2(1.2 \%)$ & $0(0 \%)$ & $<.001$ \\
\hline Severe TR & $0(0 \%)$ & $64(100 \%)$ & $3(18.8 \%)$ & $0(0 \%)$ & $0(0 \%)$ & $<.001$ \\
\hline Heart rate & $90.06 \pm 17.27$ & $93.92 \pm 19.90$ & $85.75 \pm 14.48$ & $80.58 \pm 14.47$ & $94.18 \pm 17.74$ & $<.001$ \\
\hline
\end{tabular}

All nominal data expressed as presented as $\mathrm{n}$ and percentage of total population and compared with Pearson $\chi^{2}$ or Fisher exact test. Continuous data expressed as mean \pm standard deviation with comparisons calculated with one-way analysis of variance. $L V E F$, Left ventricular ejection fraction; $R V D$, right ventricular dysfunction; $A I$, aortic valve insufficiency; $M R$, mitral regurgitation; $T R$, tricuspid regurgitation. 


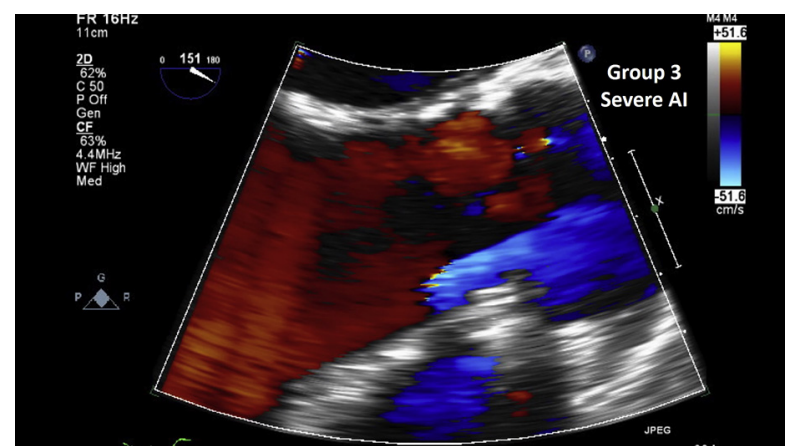

VIDEO 1. An overview of the preoperative echocardiographic categories as determined by cluster analysis and its association with subsequent clinical outcomes. It also discusses the study implications for left ventricular assist device management strategies. Video available at: https://www. jtcvs.org/article/S0022-5223(18)33246-X/fulltext.

interventions were repairs with an annuloplasty. The one mitral valve intervention was repair with an edge-to-edge Alfieri stitch placed through the LV apex.

More cfLVAD with axial design were placed in groups 1 and 2 at $61.8 \%$ and $75.0 \%$, respectively. The other groups had comparable numbers of centrifugal and axial devices. Group 2 had the lowest number of redo-sternotomies $(15.6 \%, P=.001)$ and greatest use of delayed closure of the sternum to manage coagulopathy or RVF $(60.9 \%$, $P=.002$, Table 5).

\section{Postoperative Outcomes}

There was no difference in length of intensive care unit stay, hospital stay, or days of readmission between the groups $(P>.05$, Table 5). Group 2 had the greatest incidence of postoperative $\operatorname{RVF}(20.3 \%, P=.001)$, need for RVAD use $(17.2 \%, P=.001)$, as well as the longest median duration of nitric oxide use (3 days [interquartile range 1.0], Table 5). This group also had the greatest incidence of new postoperative permanent dialysis $(6.3 \%, P=.041$, Table 5). No difference in 30-day operative mortality, LVAD survival, or survival to heart transplantation was observed among groups $(P>.05$, Table 5$)$. The Kaplan-Meier combined LVAD survival and survival to heart transplantation was also comparable $(P=.565$, Figure 1$)$.

\section{Tricuspid Valve Regurgitation and RVF}

Using regression analysis, severe TR predicted postop RVF in the total study population $(P<.001$, odds ratio [OR] 3.8) as well as in those with moderate-severe RVD $(P=.001$, OR 3.9). Despite the baseline presence of severe MR $(100 \%)$ and moderate-severe RVD (53\%) in group 1 (in the absence of severe TR), postoperative RVF was relatively low at $5.5 \%$. Group 5 had a $93.4 \%$ incidence of moderate-severe RVD at baseline with no other associated valvular lesions. Importantly, in the absence of severe TR, RVF post-cfLVAD in group 5 was also comparable at $8.8 \%$. Interestingly, in group 2 , with a baseline incidence of $100 \%$ severe TR and a significant number of patients with severe MR (70\%), the RVF rate was markedly greater at $20.3 \%$, even when taking into consideration an $80 \%$ moderate-severe RVD incidence. In group 2, the RVF rate in those with and without MR was $22.2 \%(\mathrm{n}=10 / 45)$ and $15.8 \%(\mathrm{n}=3 / 19)$, respectively $(P=.560)$. For the

TABLE 2. Operative indication, preoperative mechanical support, and operative parameters

\begin{tabular}{|c|c|c|c|c|c|c|}
\hline Cluster & $1(n=110)$ & $2(n=64)$ & $3(n=16)$ & $4(n=163)$ & $5(n=137)$ & $P$ value \\
\hline Bridge to transplant & $75(68.2 \%)$ & $50(78.1 \%)$ & $8(50 \%)$ & $85(52.1 \%)$ & $82(59.9 \%)$ & .002 \\
\hline Destination & $35(31.8 \%)$ & $14(21.9 \%)$ & $8(50 \%)$ & $78(47.9 \%)$ & $55(40.1 \%)$ & .002 \\
\hline Temporary circulatory support & $4(3.6 \%)$ & $9(14.1 \%)$ & $0(0 \%)$ & $12(7.4 \%)$ & $20(14.6 \%)$ & .012 \\
\hline $\begin{array}{l}\text { Temporary circulatory support } \\
\text { duration, } d \text {, mean }\end{array}$ & $7.75 \pm 2.75$ & $3.56 \pm 1.33$ & $0(0 \%)$ & $5.08 \pm 1.98$ & $5.70 \pm 4.00$ & .135 \\
\hline IABP & $55(50 \%)$ & $33(51.6 \%)$ & $8(50 \%)$ & $71(43.6 \%)$ & $75(54.7 \%)$ & .416 \\
\hline IABP duration, $d$, mean & $2.36 \pm 2.46$ & $2.15 \pm 2.50$ & $1.88 \pm 1.46$ & $2.25 \pm 2.38$ & $2.51 \pm 2.57$ & .922 \\
\hline Redo-sternotomy & $22(20.0 \%)$ & $10(15.6 \%)$ & $5(31.3 \%)$ & $62(38.0 \%)$ & $33(24.1 \%)$ & .001 \\
\hline Centrifugal pump & $42(38.2 \%)$ & $16(25.0 \%)$ & $8(50.0 \%)$ & $73(44.8 \%)$ & $60(43.8 \%)$ & .059 \\
\hline Axial pump & $68(61.8 \%)$ & $48(75.0 \%)$ & $8(50.0 \%)$ & $90(55.2 \%)$ & $77(56.2 \%)$ & .059 \\
\hline Cardiopulmonary bypass, min & $80.45 \pm 28.62$ & $97.91 \pm 34.44$ & $123.75 \pm 35.09$ & $79.78 \pm 33.46$ & $83.93 \pm 28.95$ & $<.001$ \\
\hline \multicolumn{7}{|l|}{ Concomitant surgery } \\
\hline Valve procedure & $45(40.9 \%)$ & $52(81.3 \%)$ & $16(100.0 \%)$ & $47(28.8 \%)$ & $56(40.9 \%)$ & $<.001$ \\
\hline $\mathrm{AV}$ procedure & $4(3.6 \%)$ & $0(0 \%)$ & $16(100.0 \%)$ & $9(5.5 \%)$ & $4(2.9 \%)$ & $<.001$ \\
\hline $\mathrm{TV}$ procedure & $39(35.5 \%)$ & $52(81.3 \%)$ & $10(62.5 \%)$ & $39(23.9 \%)$ & $53(38.7 \%)$ & $<.001$ \\
\hline Mitral valve procedure & $1(0.9 \%)$ & $0(0 \%)$ & $0(0 \%)$ & $0(0 \%)$ & $0(0 \%)$ & .484 \\
\hline
\end{tabular}

All nominal data expressed as presented as $n$ and percentage of total population and compared with Pearson $\chi^{2}$ or Fisher exact test. Continuous data expressed as mean \pm standard deviation with comparisons calculated with one-way analysis of variance. Median test was used to analyze temporary circulatory support duration and IABP duration. IABP, Intraaortic balloon pump; $A V$, aortic valve; $T V$, tricuspid valve. 
TABLE 3. Patient demographics, comorbidities, and presentation

\begin{tabular}{|c|c|c|c|c|c|c|}
\hline Cluster & $1(\mathbf{n}=110)$ & $2(n=64)$ & $3(n=16)$ & $4(n=163)$ & $5(n=137)$ & $P$ value \\
\hline Age, $y$ & $54.77 \pm 12.98$ & $51.50 \pm 15.32$ & $59.48 \pm 8.67$ & $57.50 \pm 11.94$ & $53.90 \pm 13.56$ & .010 \\
\hline Male & $82(74.5 \%)$ & $43(67.2 \%)$ & $13(81.3 \%)$ & $133(81.6 \%)$ & $113(82.5 \%)$ & .087 \\
\hline Height, $\mathrm{cm}$ & $173.72 \pm 9.58$ & $172.37 \pm 13.10$ & $173.56 \pm 10.77$ & $174.94 \pm 9.17$ & $175.57 \pm 8.89$ & .219 \\
\hline Weight, $\mathrm{kg}$ & $82.56 \pm 22.65$ & $80.99 \pm 25.61$ & $84.33 \pm 16.54$ & $88.78 \pm 20.81$ & $86.38 \pm 19.77$ & .010 \\
\hline Body mass index, $\mathrm{kg} / \mathrm{m}^{2}$ & $27.80 \pm 9.37$ & $27.05 \pm 6.61$ & $27.92 \pm 4.17$ & $28.93 \pm 6.09$ & $27.97 \pm 5.94$ & .059 \\
\hline Hypertension & $46(41.8 \%)$ & $17(26.6 \%)$ & $8(50.0 \%)$ & $99(60.7 \%)$ & $64(46.7 \%)$ & $<.001$ \\
\hline Diabetes & $33(30.0 \%)$ & $12(18.8 \%)$ & $6(37.5 \%)$ & $67(41.1 \%)$ & $49(35.8 \%)$ & .023 \\
\hline Stroke or transient ischemic attack & $10(9.1 \%)$ & $8(12.5 \%)$ & $4(25.0 \%)$ & $26(16.0 \%)$ & $12(8.8 \%)$ & .132 \\
\hline Carotid disease & $10(9.1 \%)$ & $1(1.6 \%)$ & $0(0 \%)$ & $15(9.2 \%)$ & $9(6.6 \%)$ & .201 \\
\hline Hyperlipidemia & $59(53.6 \%)$ & $26(40.6 \%)$ & $14(87.5 \%)$ & $118(72.4 \%)$ & $77(56.2 \%)$ & $<.001$ \\
\hline Atrial fibrillation & $17(15.5 \%)$ & $16(25.0 \%)$ & $5(31.3 \%)$ & $38(23.5 \%)$ & $33(24.1 \%)$ & .359 \\
\hline Dialysis & $0(0 \%)$ & $0(0 \%)$ & $0(0 \%)$ & $0(0 \%)$ & $1(0.7 \%)$ & 630 \\
\hline Implantable cardioverter defibrillator & $94(85.5 \%)$ & $55(85.9 \%)$ & $15(93.8 \%)$ & $136(83.4 \%)$ & $106(77.4 \%)$ & .265 \\
\hline Cardiac resynchronization therapy & $58(56.3 \%)$ & $32(56.1 \%)$ & $11(78.6 \%)$ & $82(50.6 \%)$ & $51(38.6 \%)$ & .008 \\
\hline \multicolumn{7}{|l|}{ INTERMACS } \\
\hline 1 & $15(13.6 \%)$ & $16(25.0 \%)$ & $1(6.3 \%)$ & $14(8.6 \%)$ & $26(19.0 \%)$ & .010 \\
\hline 2 & $37(33.6 \%)$ & $22(34.4 \%)$ & $6(37.5 \%)$ & $22(13.5 \%)$ & $42(30.7 \%)$ & $<.001$ \\
\hline 3 & $46(41.8 \%)$ & $24(37.5 \%)$ & $7(43.8 \%)$ & $99(60.7 \%)$ & $53(38.7 \%)$ & .001 \\
\hline 4 & $12(10.9 \%)$ & $2(3.1 \%)$ & $2(12.5 \%)$ & $28(17.2 \%)$ & $16(11.7 \%)$ & .064 \\
\hline
\end{tabular}

All nominal data expressed as presented as $n$ and percentage of total population and compared with Pearson $\chi^{2}$ or Fisher exact test. Continuous data expressed as mean \pm standard deviation with comparisons calculated with one-way analysis of variance. INTERMACS, Interagency Registry for Mechanically Assisted Circulatory Support.

entire study population $(\mathrm{n}=490)$, there was a trend toward severe MR being predictive for $\operatorname{RVF}(P=.229)$ and RVAD implantation $(P=.228)$, although this was not statistically significant. In group 3, characterized by severe AI, none of the patients experienced RVF despite a $62.5 \%$ incidence of preoperative moderate-severe RVD and significantly longer duration of cardiopulmonary bypass and period of cold ischemia (ie, aortic crossclamp).

Analyzing our entire patient population $(\mathrm{n}=490)$ using binary logistic regression, in patients with mild or less TR $(\mathrm{n}=271)$, neither preoperative MR grade $(P=.653)$ nor preoperative AI grade $(P=.999)$ predicted postoperative RVF. Although the presence of preoperative moderatesevere RVD predicted postoperative RVF (OR 2.17, $P<.001$ ), the additional presence of severe TR (OR 3.22, $P=.026)$ was associated with almost twice the likelihood of developing postoperative RVF compared with patients with moderate or less TR (OR 1.78, $P=.009)$. Of the patients with mild or less RVD, only 5.5\% (13/235) had severe TR. Conversely, in patients with moderate-severe RVD, 21.2\% (54/255) had severe TR. Of the patients with mild or less RVD, $4.3 \%$ had RVF and of the patients with moderate-severe RVD 11\% had RVF. In patients with severe TR $(n=67)$, the incidence of RVF in those who did and did not undergo TV repair was $16.4 \%(9 / 55)$ and $33.3 \%(4 / 12)$, respectively $(P=.178)$.

\section{DISCUSSION}

In this study, the nonhypothesis-driven statistical classification of preoperative echocardiographic findings using cluster analysis has revealed novel insights into the determinants of postoperative complications including RVF. Interestingly, the incidence of postoperative RVF $(20.3 \%)$ and RVAD use $(17.2 \%)$ was by far the greatest in group 2 , as characterized by biventricular failure and severe regurgitation of both the mitral and tricuspid valves. This group had the greatest portion of patients with bridge to transplant indication $(78 \%, P=.002)$. Conversely in group 1 , even in the presence of severe MR, the absence of significant severe TR, suggestive of lack of chronic annular remodeling due to RVD, is associated with a low incidence of RVF and RVAD use at $5.5 \%$ and $4.5 \%$, respectively.

It is possible that increased PVR from persistent MR after LVAD implant accompanied by remodeling and enlargement of the tricuspid annulus from long-standing RVD leads to predictably poor RV function after LVAD implant. Morgan and colleagues ${ }^{15}$ previously reported that LVAD implantation decreased MR severity from moderatesevere in $76 \%$ preoperatively to $8 \%$ at 6 months postoperatively. Although recent publications have improved our understanding of mitral valve pathology in the setting of LVAD implantation, there remains no consensus indication for repair of moderate-severe MR. ${ }^{16}$ Computer modeling 
TABLE 4. Preoperative hemodynamics parameters

\begin{tabular}{|c|c|c|c|c|c|c|}
\hline Cluster & $1(n=110)$ & $2(n=64)$ & $3(n=16)$ & $4(n=163)$ & $5(n=137)$ & $P$ value \\
\hline Cardiac output, L/min & $4.31 \pm 1.22$ & $4.27 \pm 1.16$ & $4.07 \pm 0.73$ & $4.77 \pm 1.25$ & $4.62 \pm 1.36$ & .006 \\
\hline Cardiac index, $\mathrm{L} / \mathrm{min} / \mathrm{m}^{2}$ & $2.20 \pm 0.55$ & $2.19 \pm 0.56$ & $1.99 \pm 0.36$ & $2.34 \pm 0.55$ & $2.30 \pm 0.65$ & .065 \\
\hline PCWP, mm Hg & $20.29 \pm 6.65$ & $20.52 \pm 6.79$ & $20.53 \pm 9.01$ & $19.06 \pm 6.59$ & $20.64 \pm 7.20$ & .309 \\
\hline PVR, wood units & $3.09 \pm 1.64$ & $2.79 \pm 1.46$ & $2.74 \pm 0.84$ & $2.63 \pm 1.33$ & $2.79 \pm 1.52$ & .275 \\
\hline Transpulmonary gradient, $\mathrm{mm} \mathrm{Hg}$ & $12.27 \pm 5.11$ & $11.01 \pm 4.55$ & $11.10 \pm 3.91$ & $11.77 \pm 5.06$ & $11.78 \pm 4.52$ & .549 \\
\hline $\begin{array}{l}\text { Systemic vascular resistance, } \\
\text { dysnes } / \mathrm{s} / \mathrm{cm}^{5}\end{array}$ & $1338.41 \pm 453.46$ & $1257.50 \pm 388.36$ & $1357.50 \pm 330.83$ & $1220.26 \pm 373.79$ & $1245.33 \pm 445.76$ & .178 \\
\hline Central venous pressure, $\mathrm{mm} \mathrm{Hg}$ & $8.40 \pm 4.45$ & $11.09 \pm 5.34$ & $8.40 \pm 5.33$ & $7.91 \pm 4.59$ & $9.69 \pm 5.35$ & $<.001$ \\
\hline RVSWI, gm $/ \mathrm{m} / \mathrm{m}^{2} /$ beat & $606.06 \pm 255.33$ & $489.32 \pm 227.98$ & $551.16 \pm 247.55$ & $673.32 \pm 271.64$ & $562.81 \pm 241.09$ & $<.001$ \\
\hline Central venous pressure/PCWP ratio & $0.43 \pm 0.21$ & $0.56 \pm 0.27$ & $0.42 \pm 0.21$ & $0.41 \pm 0.22$ & $0.47 \pm 0.25$ & $<.001$ \\
\hline $\mathrm{SVO}_{2}, \%$ & $55.13 \pm 9.28$ & $53.61 \pm 9.78$ & $54.36 \pm 7.25$ & $57.49 \pm 8.12$ & $54.89 \pm 8.58$ & .023 \\
\hline White blood count, $\times 10^{3} / \mu \mathrm{L}$ & $8.94 \pm 3.36$ & $8.67 \pm 3.04$ & $7.96 \pm 1.66$ & $8.21 \pm 2.53$ & $9.86 \pm 4.43$ & .019 \\
\hline Sodium, $\mathrm{mEq} / \mathrm{L}$ & $133.56 \pm 4.35$ & $132.36 \pm 4.85$ & $134.31 \pm 4.13$ & $134.56 \pm 4.96$ & $134.25 \pm 4.01$ & .035 \\
\hline Bicarbonate, $\mathrm{mEq} / \mathrm{L}$ & $27.99 \pm 3.80$ & $27.30 \pm 4.26$ & $27.50 \pm 5.19$ & $27.83 \pm 3.81$ & $28.56 \pm 3.90$ & .255 \\
\hline Blood urea nitrogen, $\mathrm{mg} / \mathrm{dL}$ & $29.01 \pm 12.13$ & $31.48 \pm 18.31$ & $31.56 \pm 15.33$ & $30.49 \pm 14.19$ & $32.85 \pm 16.06$ & .364 \\
\hline Creatinine, $\mathrm{mg} / \mathrm{dL}$ & $1.28 \pm 0.37$ & $1.32 \pm 0.53$ & $1.48 \pm 0.48$ & $1.36 \pm 0.60$ & $1.30 \pm 0.46$ & .485 \\
\hline Alkaline phosphatase, IU/L & $99.13 \pm 45.19$ & $108.33 \pm 44.42$ & $94.81 \pm 35.34$ & $94.13 \pm 44.58$ & $107.85 \pm 56.57$ & .010 \\
\hline Total bilirubin, $\mathrm{mg} / \mathrm{dL}$ & $1.23 \pm 0.70$ & $1.50 \pm 1.02$ & $1.19 \pm 0.61$ & $0.93 \pm 0.55$ & $1.33 \pm 1.09$ & $<.001$ \\
\hline Brain natriuretic peptide, $\mathrm{pg} / \mathrm{mL}$ & $1002.60 \pm 988.17$ & $1156.60 \pm 1047.45$ & $1026.53 \pm 839.00$ & $604.12 \pm 699.57$ & $1134.87 \pm 1214.68$ & $<.001$ \\
\hline
\end{tabular}

Continuous data expressed as mean \pm standard deviation with comparisons calculated with one-way analysis of variance. PCWP, Pulmonary capillary wedge pressure; $P V R$, pulmonary vascular resistance; $R V S W I$, right ventricular stroke work index; $\mathrm{SVO}_{2}$, mixed venous oxygen saturation.

demonstrated that at LVAD speeds that allow AV opening, moderate-severe MR can cause a significant increase in left atrial and pulmonary artery pressures. ${ }^{17}$ It is worth noting that mitral valve repair may lead to greater reductions in PVR and increase the likelihood of bridge to transplantation. This may also lower the incidence of heart failure related readmissions. ${ }^{18}$

A recent study by Robertson and colleagues ${ }^{19}$ demonstrated a decreased rate of readmission and an improved quality of life in patients who had mitral valve intervention at the time of LVAD implantation. Although our results demonstrated a trend $(P=.2)$ toward severe MR being predictive for postoperative RVF and RVAD in the immediate postoperative period, this may hold greater implications for long-term outcomes. A larger study with greater power may clarify this issue. Furthermore, it may be post-LVAD implant MR severity, not preoperative MR severity, that impacts RV function in addition to native RV function and contributions by other valvular pathologies.

Group 3 was characterized by severe AI and had a $62.5 \%$ incidence of moderate-severe RVD as well as $38.9 \%$ with severe MR. Although many studies have focused on newonset $\mathrm{AI}$ after prolonged cfLVAD support, ${ }^{20}$ the implications of preoperative AI are less well understood. Our data show that preoperative RVD in this setting rarely translates into severe RVD after LVAD implant, as illustrated by a $0 \%$ incidence of RVF and RVAD when not accompanied by moderate-severe TR. It is also likely that mechanical circulatory support was contraindicated in the presence of severe $\mathrm{AI}$ and lead to this group undergoing early cfLVAD implantation. Our results show that end-stage heart failure in this group is relatively well compensated and the right ventricle responds well to a decrease in LVEDP after AV surgery and LVAD implantation. Interestingly, this group had the oldest age $(P=.010)$, the largest portion of these patients who received cardiac resynchronization therapy $(78.6 \%, P=.008)$, and the lowest proportion of patients classified as INTERMACS 1 at the time of LVAD implantation $(6.3 \%, P=.010)$.

In group 4, which had isolated LV dysfunction in the presence of predominantly mild or less RVD and without valvular disease, the proportion presenting in INTERMACS 1 was relatively low at $8.6 \%$ and brain natriuretic peptide was the lowest at $604.1 \pm 699.6(P<.001)$. Preserved RV function can be seen with the lowest central venous pressure $(7.9 \pm 4.6, P<.001)$ and greatest RVSWI $673.3 \pm 271.6$ $(P<.001)$. Postoperative RVF was also relatively low at $4.3 \%$. Therefore, in the absence of valvular pathology and relatively preserved RV function preoperatively, the incidence of RVF will be predictably low post-LVAD.

Group 5 was characterized by $93.4 \%$ moderate-severe RVD but a lack of valvular pathology. A significant 
TABLE 5. Postoperative outcomes

\begin{tabular}{|c|c|c|c|c|c|c|}
\hline Cluster & $1(n=110)$ & $2(n=64)$ & $3(n=16)$ & $4(n=163)$ & $5(\mathbf{n}=137)$ & $P$ value \\
\hline Total intensive care unit LOS, $d$, median & 7.0 (IQR 5.0) & 8.0 (IQR 12.0) & 7.0 (IQR 10.0) & 7.0 (IQR 6.0) & 7.0 (IQR 6.0) & .702 \\
\hline Total LOS, d, median & 20.5 (IQR 13.0) & 25.0 (IQR 13.0) & 19.5 (IQR 19.0) & $21.0($ IQR 13.0$)$ & $21.0($ IQR 14.0$)$ & .556 \\
\hline Total days of readmission, median & $23.0(\mathrm{IQR} 44.0)$ & 20.5 (IQR 60.0) & 47.5 (IQR 83.0) & 26.0 (IQR 79.0) & 20.0 (IQR 52.0) & .268 \\
\hline Number of readmissions, median & 3.0 (IQR 4.0) & 3.0 (IQR 5.0) & $6(\mathrm{IQR} 8.0)$ & 3.0 (IQR 9.0) & 2.0 (IQR 5.0) & .197 \\
\hline RV failure & $6(5.5 \%)$ & $13(20.3 \%)$ & $0(0 \%)$ & $7(4.3 \%)$ & $12(8.8 \%)$ & .001 \\
\hline RVAD & $5(4.5 \%)$ & $11(17.2 \%)$ & $0(0 \%)$ & $5(3.1 \%)$ & $9(6.6 \%)$ & .001 \\
\hline Concurrent LVAD/RVAD & $5(100.0 \%)$ & $10(90.9 \%)$ & $0(0 \%)$ & $3(60.0 \%)$ & $9(100.0 \%)$ & .087 \\
\hline Delayed unplanned RVAD & $0(0.0 \%)$ & $1(9.1 \%)$ & $0(0 \%)$ & $2(40.0 \%)$ & $0(0.0 \%)$ & .087 \\
\hline RVAD duration, $\mathrm{d}$, median & 45.5 (IQR 118) & 13.0 (IQR 52.0) & $0(0 \%)$ & 17.0 (IQR 90.0) & 17.0 (IQR 19.0) & .426 \\
\hline RVAD duration, $\mathrm{d}$, median & $57.75 \pm 62.73$ & $34.0 \pm 46.17$ & $43.0 \pm 46.17$ & $43.0 \pm 68.22$ & $19.29 \pm 13.61$ & .622 \\
\hline Nitric oxide use & $108(98.2 \%)$ & $63(98.4 \%)$ & $16(100 \%)$ & $159(97.5 \%)$ & $136(99.3 \%)$ & .796 \\
\hline Nitric oxide duration, $\mathrm{d}$, median & 2.0 (IQR 1.0) & 3.0 (IQR 1.0) & 2.0 (IQR 1.0) & $2.0($ IQR 1.0$)$ & 2.0 (IQR 2.0) & .001 \\
\hline Delayed sternal closure & $42(38.2 \%)$ & $39(60.9 \%)$ & $9(56.3 \%)$ & $54(33.1 \%)$ & $50(36.5 \%)$ & .002 \\
\hline Chest open days, mean & $1.43 \pm 0.83$ & $1.56 \pm 0.91$ & $1.11 \pm 0.33$ & $1.37 \pm 0.65$ & $1.30 \pm 0.61$ & .358 \\
\hline Device infection & $25(22.7 \%)$ & $22(34.4 \%)$ & $5(31.3 \%)$ & $42(25.8 \%)$ & $27(19.7 \%)$ & .218 \\
\hline Device exchange infection & $6(5.5 \%)$ & $8(12.5 \%)$ & $0(0 \%)$ & $9(5.5 \%)$ & $5(3.6 \%)$ & .107 \\
\hline Late $\mathrm{AV}$ intervention & $1(0.9 \%)$ & $0(0 \%)$ & $0(0 \%)$ & $1(0.6 \%)$ & $4(2.9 \%)$ & .304 \\
\hline All stroke & $27(24.5 \%)$ & $12(18.8 \%)$ & $3(18.8 \%)$ & $39(23.9 \%)$ & $22(16.1 \%)$ & .415 \\
\hline Hemorrhagic stroke & $13(11.8 \%)$ & $6(9.4 \%)$ & $2(12.5 \%)$ & $21(12.9 \%)$ & $10(7.3 \%)$ & .591 \\
\hline Embolic stroke & $14(12.7 \%)$ & $6(9.4 \%)$ & $1(6.3 \%)$ & $18(11.0 \%)$ & $12(8.8 \%)$ & .831 \\
\hline Hemolysis & $25(22.7 \%)$ & $14(21.9 \%)$ & $4(25.0 \%)$ & $39(23.9 \%)$ & $33(24.1 \%)$ & .996 \\
\hline Postoperative dialysis & $4(3.6 \%)$ & $6(9.4 \%)$ & $1(6.3 \%)$ & $7(4.3 \%)$ & $5(3.6 \%)$ & .422 \\
\hline Postoperative permanent dialysis & $3(2.7 \%)$ & $4(6.3 \%)$ & $0(0 \%)$ & $1(0.6 \%)$ & $1(0.7 \%)$ & .041 \\
\hline Reoperation for bleeding & $11(10.0 \%)$ & $7(10.9 \%)$ & $3(18.8 \%)$ & $14(8.6 \%)$ & $11(8.0 \%)$ & .607 \\
\hline $\begin{array}{l}\text { Operative mortality (30-d or } \\
\text { in-hospital) }\end{array}$ & $5(4.5 \%)$ & $6(9.4 \%)$ & $0(0 \%)$ & $8(4.9 \%)$ & $5(3.6 \%)$ & .397 \\
\hline cfLVAD death (cumulative) & $32(29.1 \%)$ & $21(32.8 \%)$ & $4(25.0 \%)$ & $53(32.5 \%)$ & $33(24.1 \%)$ & .535 \\
\hline Heart transplant & $46(41.8 \%)$ & $25(39.1 \%)$ & $6(37.5 \%)$ & $50(30.7 \%)$ & $47(34.3 \%)$ & .400 \\
\hline
\end{tabular}

All nominal data expressed as presented as $n$ and percentage of total population and compared with Pearson $\chi^{2}$ or Fisher exact test. Continuous data expressed as mean \pm standard deviation with comparisons calculated with one-way analysis of variance. Median test was used to analyze total days of readmission, RVAD duration, nitric oxide duration, and chest open days. $L O S$, Length of stay; $I Q R$, interquartile range; $R V$, right ventricle; $R V A D$, right ventricular assist device; $L V A D$, left ventricular assist device; $A V$, aortic valve; cfLVAD, continuous-flow left ventricular assist device.

proportion in this group needed temporary circulatory support preoperatively $(14.6 \%)$. This group had a postoperative RVF rate of $8.8 \%$, but was much lower compared with group $2(20.3 \%, P=.021)$. In the absence of MR and TR, the incidence of RVF remains relatively low even in the presence of biventricular failure. This finding reinforces the important role of TR and RV remodeling in the prediction of postoperative RVF during LVAD implant.

The presence of both severe TR and RVD is highly predictive of RVF with an OR of $3.22(P=.026)$. Moderatesevere RVD alone with moderate or less TR is a much weaker RVF predictor with an OR $1.78(P=.009)$. This association is further strengthened if the patient echo profile conforms to cluster number 2 with biventricular failure and severe MR. An interpretation of these findings is that if severe TR persists after diuresis and medical optimization, then the TR is likely reflective of chronic tricuspid annular remodeling by enlargement due to long standing RVD rather than acute volume overload. Indeed, TR in patients with heart failure was previously demonstrated to be associated with tricuspid annular dilatation and RV enlargement with apical leaflet displacement, which is associated with RVF after LVAD implantation. ${ }^{21,22}$ Piacentino and colleagues $^{21}$ found that at late follow-up after LVAD implant (mean $156 \pm 272$ days), the incidence of moderate-severe TR deceased from $49 \%$ to $32 \%$, consistent with slow and incomplete RV remodeling. Although a survival benefit for TV repair during LVAD implantation was demonstrated by some investigators, ${ }^{23}$ others did not demonstrate a positive impact on survival. ${ }^{24}$ 


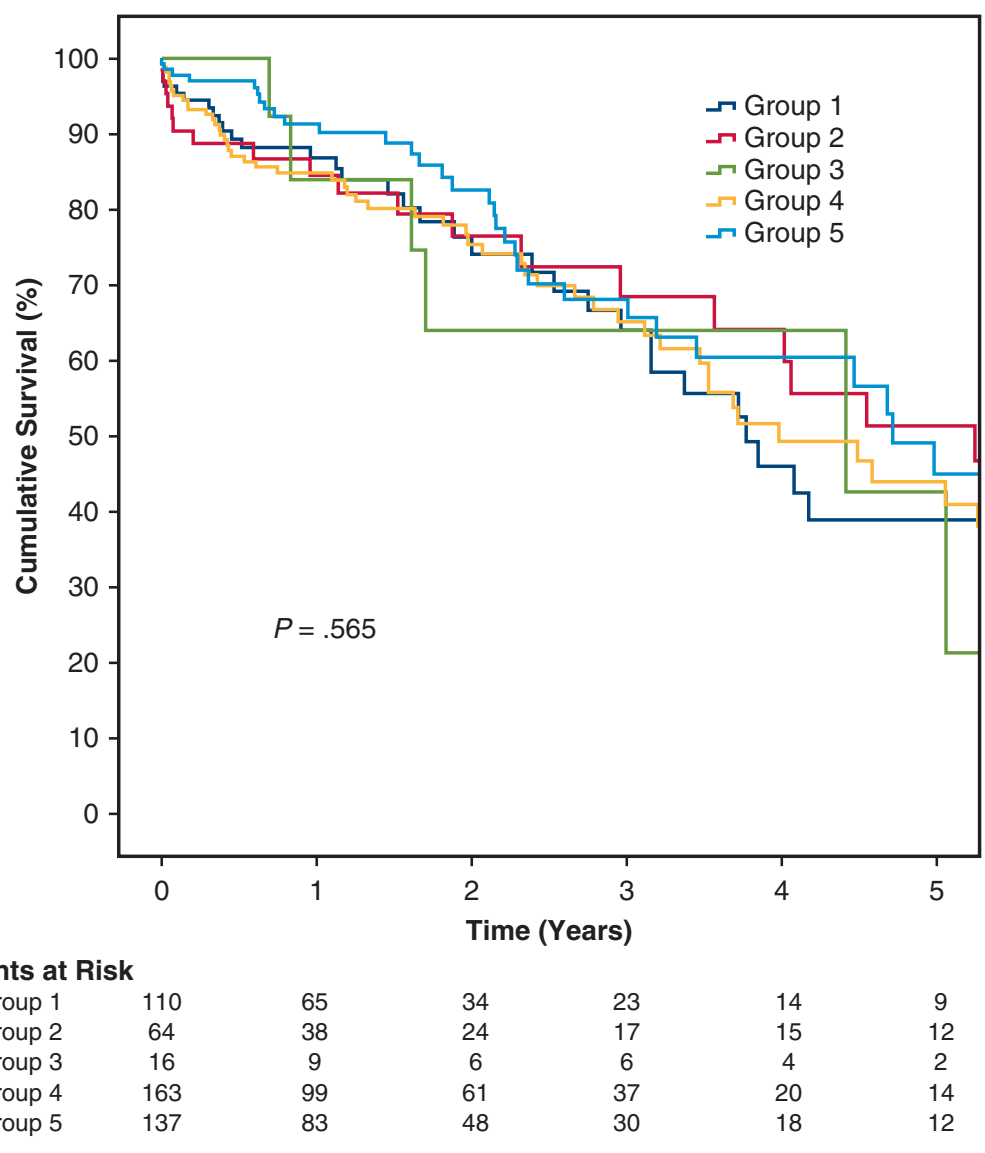

A

\begin{tabular}{|l|c|c|c|c|c|}
\hline Follow Up Years & $\mathbf{1}$ & $\mathbf{2}$ & $\mathbf{3}$ & $\mathbf{4}$ & $\mathbf{5}$ \\
\hline Group 1 & $87.8(79.4 ; 92.9)$ & $77.0(65.1 ; 85.3)$ & $64.7(50.0 ; 76.0)$ & $46.6(30.7 ; 61.1)$ & $39.4(23.7 ; 54.8)$ \\
\hline Group 2 & $85.9(73.5 ; 92.7)$ & $77.7(62.6 ; 87.3)$ & $69.5(51.4 ; 82.0)$ & $65.2(46.1 ; 78.9)$ & $52.1(32.3 ; 68.7)$ \\
\hline Group 3 & $82.5(46.1 ; 95.3)$ & $72.2(35.7 ; 90.2)$ & $72.2(35.7 ; 90.2)$ & $72.2(35.7 ; 90.2)$ & $48.1(8.6 ; 80.4)$ \\
\hline Group 4 & $86.6(79.9 ; 91.2)$ & $76.2(67.4 ; 83.0)$ & $63.2(52.2 ; 72.4)$ & $48.6(35.9 ; 60.2)$ & $43.1(29.8 ; 55.6)$ \\
\hline Group 5 & $91.2(84.2 ; 95.2)$ & $82.3(72.0 ; 89.0)$ & $71.2(58.6 ; 80.6)$ & $61.2(46.9 ; 72.8)$ & $46.6(30.1 ; 61.4)$ \\
\hline B
\end{tabular}

FIGURE 1. Kaplan-Meier survival plot for cluster categories. A, There was no difference in combined left ventricular assist device survival and survival to heart transplantation between the 5 cluster groups. Comparable survival outcomes can be achieved despite varying degrees of preoperative valvular pathology and ventricular dysfunction. B, Survival proportion ( $\%$ ) with $95 \%$ confidence intervals for each group at 1, 2, 3, 4, and 5 years' follow-up is presented. For the study population the 1-, 3-, and 5-year survival was $87.1 \%, 66.2 \%$, and $44.4 \%$, respectively.

Whether severe TR is simply a marker of significant underlying RVD versus having an independent role in worsening forward right-sided cardiac output remains unclear. Furthermore, TR can mask underlying RVD, leading to worsened apparently RV function after re-establishing TV competence. ${ }^{25}$ We did identify a small population of patients with mild or less RVD but severe TR $(\mathrm{n}=13)$. It is possible that underlying RV myocardial contractility in this group was overestimated on echo due to the presence of severe TR and decompression into a low pressure central venous system. Our present study suggests that multivalvular interactions may be important to predict RVF. TV repair in severe TR may not effectively improve RV performance if significant MR remains after LVAD implantation. Residual severe MR will negatively impact RV performance by increasing pulmonary artery pressures and resistance.

An improved ability to identify patients at risk for RVF will facilitate early or simultaneous use of RVAD with cfLVAD implantation. This is important, given the improved 180 days survival for patients who received an 


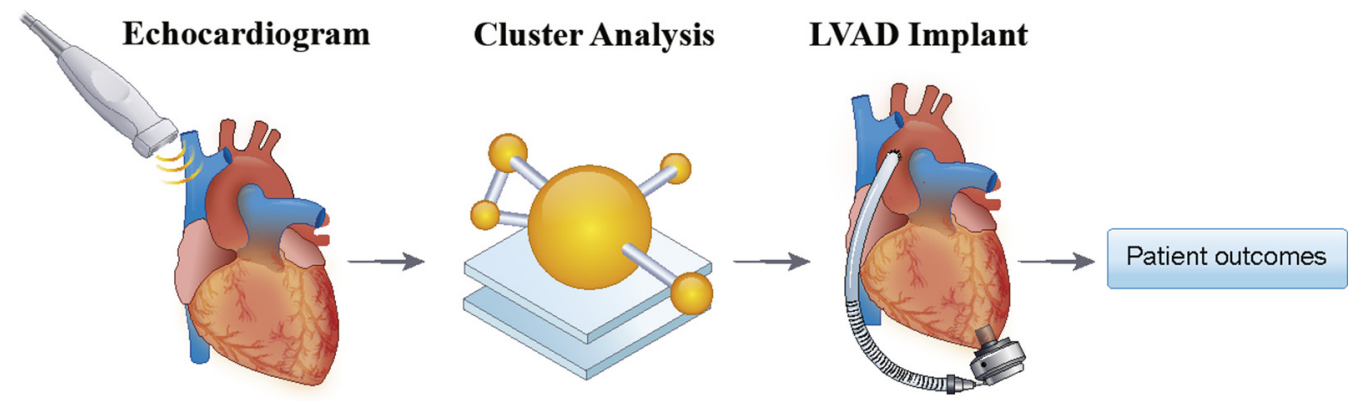

FIGURE 2. Cluster analysis of preoperative echocardiographic findings and association with outcomes after LVAD implantation. Shown is a graphical depiction of the study design using cluster analysis to categorize preoperative echocardiographic findings. The segregated groups have implications for postoperative course after LVAD implantation. LVAD, Left ventricular assist device.

RVAD within 24 hours of LVAD surgery rather than adopting a strategy of watchful waiting. ${ }^{3}$ Kapelios and colleagues $^{26}$ also describes a syndrome of late-onset RVF during LVAD support where RVF can manifest several months to years from device implantation with adverse prognostic implications in terms of mortality and survival to heart transplantation. However, as our LVAD survival and survival to heart transplantation data show, comparable long-term outcomes can be achieved using modern mechanical circulatory support strategies.

Presently, our practice is that severe degrees of TR was nearly all uniformly treated with TV repair. The decision for TV repair has been influenced by surgeon preference, and treatment of lesser degrees of TR has been evolving over time. MR was almost always not repaired even under circumstances of severe MR. Severe AI is uniformly addressed intraoperatively although treatment of AI has also been evolving to address lesser degrees of $\mathrm{AI}$ at the time of LVAD implant, which is also influenced by surgeon preference.

This study is limited by the retrospective single-center design with inherent biases. Ventricular contractility is load-dependent and may vary between echocardiographic assessment at different times. RVF was not defined by a quantitative parameter of RV contractility, which leads to a degree of subjectivity. Detailed echocardiographic data regarding RV function (eg, tricuspid annular plane systolic excursion, RV dimension, RV ejection fraction) were not available. Concomitant valvular procedures may have influenced outcomes. Although we did not find a statistically significant impact on RVF by TV repair in the setting of severe TR $(P=.178)$, our study may not be sufficiently powered to demonstrate an effect and TR severity may be dynamic. The lack of difference in survival can be explained by the fact the group 2 received further post-implant treatments for RVF (eg, pulmonary vasodilators, RVAD) that lead to improved hemodynamics sufficiently for bridge to transplantation or destination therapy. Our study did not capture outpatients who return with mild RVF symptoms, thus underestimating the burden of late RVF. RVF postcfLVAD can also be precipitated by other causes, including acute postoperative pulmonary hypertension (eg, hypoxia) or impairment of RV function from inadequate myocardial preservation. ${ }^{27}$ Because of the limited patient population, we were not able to include cfLVAD device type in our analyses.

\section{CONCLUSIONS}

Our study uses an unsupervised nonhypothesis-driven statistical clustering methodology that reveals a grouping of echocardiographic findings that predict postoperative outcomes after cfLVAD implant (Figure 2). Moderatesevere RVD, severe TR, as well as severe MR portend a high risk of RVF. In particular, severe TR is an important marker for postoperative RVF. Despite the diverse cardiac morphologies and dysfunction leading to LVAD implant, comparable LVAD survival and bridge to transplant can be achieved.

\section{Conflict of Interest Statement}

Dr Keith Aaronson has received grants from Medtronic, and Abbott as well as received personal fees from Medtronic, Procyrion, and NuPulseCV. Other authors have nothing to disclose with regard to commercial support.

\section{References}

1. Mehra MR, Naka Y, Uriel N, Goldstein DJ, Cleveland JC Jr, Colombo PC, et al. A fully magnetically levitated circulatory pump for advanced heart failure. $N$ Engl $J$ Med. 2017:376:440-50.

2. Rogers JG, Pagani FD, Tatooles AJ, Bhat G, Slaughter MS, Birks EJ, et al. Intrapericardial left ventricular assist device for advanced heart failure. $N$ Engl J Med. 2017;376:451-60.

3. Kormos RL, Teuteberg JJ, Pagani FD, Russell SD, John R, Miller LW, et al. Right ventricular failure in patients with the HeartMate II continuous-flow left ventricular assist device: incidence, risk factors, and effect on outcomes. J Thorac Cardiovasc Surg. 2010;139:1316-24.

4. Yoshioka D, Takayama H, Garan RA, Topkara VK, Han J, Kurlansky P, et al. Contemporary outcome of unplanned right ventricular assist device for severe right heart failure after continuous-flow left ventricular assist device insertion. Interact Cardiovasc Thorac Surg. 2017;24:828-34.

5. Takeda K, Naka Y, Yang JA, Uriel N, Colombo PC, Jorde UP, et al. Outcome of unplanned right ventricular assist device support for severe right heart failure 
after implantable left ventricular assist device insertion. J Heart Lung Transplant. 2014;33:141-8.

6. Kalogeropoulos AP, Kelkar A, Weinberger JF, Morris AA, Georgiopoulou VV, Markham DW, et al. Validation of clinical scores for right ventricular failure prediction after implantation of continuous-flow left ventricular assist devices. $J$ Heart Lung Transplant. 2015;34:1595-603.

7. Wang TS, Hernandez AF, Felker GM, Milano CA, Rogers JG, Patel CB. Valvular heart disease in patients supported with left ventricular assist devices. Circ Heart Fail. 2014;7:215-22.

8. Taghavi S, Hamad E, Wilson L, Clark R, Jayarajan SN, Uriel N, et al. Mitral valve repair at the time of continuous-flow left ventricular assist device implantation confers meaningful decrement in pulmonary vascular resistance. ASAIO J. 2013;59:469-73.

9. Cowger JA, Aaronson KD, Romano MA, Haft J, Pagani FD. Consequences of aortic insufficiency during long-term axial continuous-flow left ventricular assist device support. J Heart Lung Transplant. 2014;33:1233-40.

10. Topilsky Y, Oh JK, Shah DK, Boilson BA, Schirger JA, Kushwaha SS, et al. Echocardiographic predictors of adverse outcomes after continuous left ventricular assist device implantation. JACC Cardiovasc Imaging. 2011:4:211-22.

11. Kent P, Jensen RK, Kongsted A. A comparison of three clustering methods for finding subgroups in MRI, SMS or clinical data: SPSS TwoStep Cluster analysis, Latent Gold and SNOB. BMC Med Res Methodol. 2014;14:113.

12. Dunson DB, Chen Z, Harry JA. Bayesian approach for joint modeling of cluster size and subunit-specific outcomes. Biometrics. 2003;59:521-30.

13. Chiu T, Fang D, Chen J, Wang Y, Jeris C. A Robust and Scalable Clustering Algorithm for Mixed Type Attributes in Large Database Environment. San Francisco, Calif: Proceedings of the Seventh ACM SIGKDD International Conference on Knowledge Discovery and Data Mining; 2001. 263-8. 502549: ACM.

14. Fraley C, Raftery AE. How many clusters? Which clustering method? Answers via model-based cluster analysis. Computer J. 1998;41:578-88.

15. Morgan JA, Brewer RJ, Nemeh HW, Murthy R, Williams CT, Lanfear DE, et al Left ventricular reverse remodeling with a continuous flow left ventricular assist device measured by left ventricular end-diastolic dimensions and severity of mitral regurgitation. ASAIO J. 2012;58:574-7.

16. Feldman D, Pamboukian SV, Teuteberg JJ, Birks E, Lietz K, Moore SA, et al. The 2013 International Society for Heart and Lung Transplantation guidelines for mechanical circulatory support: executive summary. J Heart Lung Transplant. 2013; 32:157-87.

17. Jelenc M, Jelenc B, Vrtovec B, Knezevic I. Mitral regurgitation and axial flow left ventricular assist device: a computer simulation study. ASAIO J. 2013;59:405-9.
18. Sandoval E, Singh SK, Carillo JA, Baldwin ACW, Ono M, Anand J, et al. Impact of concomitant mitral valve repair for severe mitral regurgitation at the time of continuous-flow left ventricular assist device insertion. Interact Cardiovasc Thorac Surg. 2017;25:620-3.

19. Robertson JO, Naftel DC, Myers SL, Tedford RJ, Joseph SM, Kirklin JK, et al. Concomitant mitral valve procedures in patients undergoing implantation of continuous-flow left ventricular assist devices: an INTERMACS database analysis. J Heart Lung Transplant. 2018;37:79-88.

20. Rajagopal K, Daneshmand MA, Patel CB, Ganapathi AM, Schechter MA, Rogers JG, et al. Natural history and clinical effect of aortic valve regurgitation after left ventricular assist device implantation. J Thorac Cardiovasc Surg. 2013; 145:1373-9.

21. Piacentino V III, Williams ML, Depp T, Garcia-Huerta K, Blue L, Lodge AJ, et al. Impact of tricuspid valve regurgitation in patients treated with implantable left ventricular assist devices. Ann Thorac Surg. 2011;91:1342-6; discussion 6-7.

22. Potapov EV, Stepanenko A, Dandel M, Kukucka M, Lehmkuhl HB, Weng Y, et al. Tricuspid incompetence and geometry of the right ventricle as predictors of right ventricular function after implantation of a left ventricular assist device. J Heart Lung Transplant. 2008;27:1275-81.

23. El Atrache M, Brewer R, Nemeh H, Williams C, Tita C, Paone G, et al. Tricuspid repair at the time of lvad implantation is associated with improved survival. J Am Coll Cardiol. 2012;59:E881.

24. Maltais S, Topilsky Y, Tchantchaleishvili V, McKellar SH, Durham LA, Joyce LD, et al. Surgical treatment of tricuspid valve insufficiency promotes early reverse remodeling in patients with axial-flow left ventricular assist devices. J Thorac Cardiovasc Surg. 2012;143:1370-6.

25. Lella LK, Sales VL, Goldsmith Y, Chan J, Iskandir M, Gulkarov I, et al. Reduced right ventricular function predicts long-term cardiac re-hospitalization after cardiac surgery. PLoS One. 2015;10:e0132808.

26. Kapelios CJ, Charitos C, Kaldara E, Malliaras K, Nana E, Pantsios C, et al. Lateonset right ventricular dysfunction after mechanical support by a continuous-flow left ventricular assist device. J Heart Lung Transplant. 2015;34:1604-10.

27. Lee S, Kamdar F, Madlon-Kay R, Boyle A, Colvin-Adams M, Pritzker M, et al Effects of the HeartMate II continuous-flow left ventricular assist device on right ventricular function. J Heart Lung Transplant. 2010;29:209-15.

Key Words: cluster analysis, left ventricular assist device, mitral regurgitation, tricuspid regurgitation, right ventricular failure, echocardiography 
TABLE E1. Demographics of patient population

\begin{tabular}{lc}
\hline & $\mathbf{N}=\mathbf{4 9 0}$ \\
\hline Age, y & $55.16 \pm 13.1$ \\
Male & $284(78.4 \%)$ \\
Height, cm & $174.46 \pm 9.85$ \\
Weight, kg & $85.55 \pm 21.63$ \\
Body mass index & $28.13 \pm 6.95$ \\
Hypertension & $234(47.8 \%)$ \\
\hline Diabetes & $167(34.1 \%)$ \\
Stroke or transient ischemic attack & $60(12.2 \%)$ \\
Carotid disease & $35(7.1 \%)$ \\
Hyperlipidemia & $294(60.0 \%)$ \\
Atrial fibrillation & $109(22.2 \%)$ \\
Dialysis & $1(0.2 \%)$ \\
\hline INTERMACS & \\
$\quad 1$ & $72(14.7 \%)$ \\
2 & $129(26.3 \%)$ \\
3 & $229(46.7 \%)$ \\
4 & $60(12.2 \%)$ \\
\hline All nominal data expressed as presented as n and percentage of total population and \\
continuous data expressed as mean \pm standard deviation. INTERMACS, Interagency \\
Registry for Mechanically Assisted Circulatory Support.
\end{tabular}

TABLE E2. Hemodynamics and preoperative echocardiographic findings in the patient population

\begin{tabular}{|c|c|}
\hline & $\mathbf{N}=490$ \\
\hline Cardiac output, L/min & $4.54 \pm 1.27$ \\
\hline Cardiac index, $\mathrm{L} / \mathrm{min} / \mathrm{m}^{2}$ & $2.27 \pm 0.58$ \\
\hline PCWP, mm Hg & $20.02 \pm 6.89$ \\
\hline PVR, wood units & $2.80 \pm 1.47$ \\
\hline Transpulmonary gradient, $\mathrm{mm} \mathrm{Hg}$ & $11.76 \pm 4.83$ \\
\hline Systemic vascular resistance, dysnes $/ \mathrm{s} / \mathrm{cm}^{5}$ & $1262.88 \pm 415.23$ \\
\hline Central venous pressure, $\mathrm{mm} \mathrm{Hg}$ & $8.95 \pm 5.01$ \\
\hline RVSWI, $g / \mathrm{m} / \mathrm{m}^{2} /$ beat & $599.25 \pm 259.96$ \\
\hline Central venous pressure/PCWP ratio & $0.45 \pm 0.23$ \\
\hline $\mathrm{SVO}_{2}, \%$ & $55.63 \pm 8.80$ \\
\hline White blood count, $\times 10^{3} / \mu \mathrm{L}$ & $8.89 \pm 3.44$ \\
\hline Sodium, $\mathrm{mEq} / \mathrm{L}$ & $133.95 \pm 4.57$ \\
\hline Bicarbonate, $\mathrm{mEq} / \mathrm{L}$ & $27.99 \pm 3.95$ \\
\hline Blood urea nitrogen, $\mathrm{mg} / \mathrm{dL}$ & $30.99 \pm 14.95$ \\
\hline Creatinine, $\mathrm{mg} / \mathrm{dL}$ & $1.33 \pm 0.51$ \\
\hline Alkaline phosphatase, IU/L & $100.98 \pm 48.32$ \\
\hline Total bilirubin, mg/dL & $1.19 \pm 0.85$ \\
\hline Brain natriuretic peptide, $\mathrm{pg} / \mathrm{mL}$ & $930.16 \pm 1005.92$ \\
\hline Lactic acid, mg/dL & $1.32 \pm 2.34$ \\
\hline LVEF, \% & $15.36 \pm 5.72$ \\
\hline Moderate-severe RVF & $255(52.0 \%)$ \\
\hline Severe AI & $16(3.3 \%)$ \\
\hline Severe MR & $163(33.3 \%)$ \\
\hline Severe TR & $67(13.7 \%)$ \\
\hline Heart rate & $88.42 \pm 17.76$ \\
\hline \multicolumn{2}{|c|}{$\begin{array}{l}\text { All nominal data expressed as presented as n and percentage of total population and } \\
\text { continuous data expressed as mean } \pm \text { standard deviation. } P C W P \text {, Pulmonary capillary } \\
\text { wedge pressure; } P V R \text {, pulmonary vascular resistance; } R V S W I \text {, right ventricular stroke } \\
\text { work index; } S V O_{2} \text {, mixed venous oxygen saturation; } L V E F \text {, left ventricular ejection } \\
\text { fraction; } R V F \text {, right ventricular failure; } A I \text {, aortic insufficiency; } M R \text {, mitral regurgita- } \\
\text { tion; } T R \text {, tricuspid regurgitation. }\end{array}$} \\
\hline
\end{tabular}

